\title{
Keratoconus-like tomographic changes in a case of recurrent interstitial keratitis
}

\author{
Marie-Sophie Hanet ${ }^{1 *}$ (D, Annette Zimpfer ${ }^{2}$, Sabine Lepper ${ }^{1}$ and Berthold Seitz
}

\begin{abstract}
Background: The purpose of this report was to describe a single case of recurrent interstitial keratitis in a young patient leading to keratoconus-like corneal changes.

Results: Over the 2 years follow-up, the patient developed clinical signs of keratoconus with Vogt's striae, Fleischer Ring and paracentral stromal thinning in the affected eye only. The tomographic measurements revealed a rapid reduction of corneal thickness from 581 to $303 \mu \mathrm{m}$ and an irregular steepening of the pathological cornea. True net corneal power increased from 42.8 to 48.8 dioptres (D) and maximal power of the anterior surface from 44.9 to 66.9 D. Best-corrected visual acuity (BCVA) decreased from 20/20 to 20/200. A penetrating excimer laser keratoplasty was performed to restore vision and corneal stability.
\end{abstract}

Conclusion: Keratoconus-like changes can occur in young patients with recurrent interstitial keratitis and request corneal transplantation.

Keywords: Keratoconus, Interstitial keratitis, Inflammation

\section{Findings}

\section{Introduction}

Keratoconus is a degenerative disorder of the cornea associated with corneal thinning and ectasia that leads to refractive errors and impaired vision. It is mostly bilateral, typically affects adolescents or young adults and may progress until the third or fourth decade of life, when it usually arrests [1].

The pathophysiology of keratoconus is complex and still remains unclear.

Recent studies revealed the probable influence of inflammatory mechanisms in the pathogenesis of keratoconus and emphasises the importance of controlling inflammation to prevent progression of the disease [2-4].

Infectious keratitis can cause topographic changes resulting in visual impairment. This phenomenon was recently investigated, and tomographic measurements of post-infected corneas revealed different patterns of corneal aberrations including protrusion keratoconus-like patterns $[5,6]$.

\footnotetext{
* Correspondence: msophiehanet@hotmail.com

${ }^{1}$ Department of Ophthalmology, Saarland University Medical Center, 66424

Homburg, Saar, Germany

Full list of author information is available at the end of the article
}

In support of these considerations, we report the case of a young patient with clinical manifestations of corneal inflammation, who developed strictly unilateral keratoconus-like changes in the affected eye.

\section{Case report}

A 27-year-old Caucasian man was referred to our center with complaints of persistent blurred vision on the left eye. He was treated with corticoid eye drops and systemic acyclovir tablets for the past 2 months on the suspicion of herpetic keratitis. No other ophthalmological pathology or systemic disease was documented.

On initial presentation, he had a best-corrected visual acuity (BCVA) of 20/20 in both eyes. His refraction was -3.25 sphere $/-0.50$ cylinder at $180^{\circ}$ in the right eye and -3.50 sphere/ -1.00 cylinder at $175^{\circ}$ in the left eye. The slit lamp examination revealed a hazy opacification of the deep stroma in the inferior nasal part of the left cornea with endothelial deposits (Fig. 1). Tomographic measurements (Oculus-Pentacam HR) revealed maximal anterior, posterior and mean total keratometry values from $44.9,-6.1 \mathrm{D}$ and $42.8 \mathrm{D}$ respectively, and a minimal corneal thickness of $581 \mu \mathrm{m}$ on the left eye. Optical coherence tomography (Casia SS-100, Tomey) showed a deep stromal hyperreflexity in the corneal center of the 


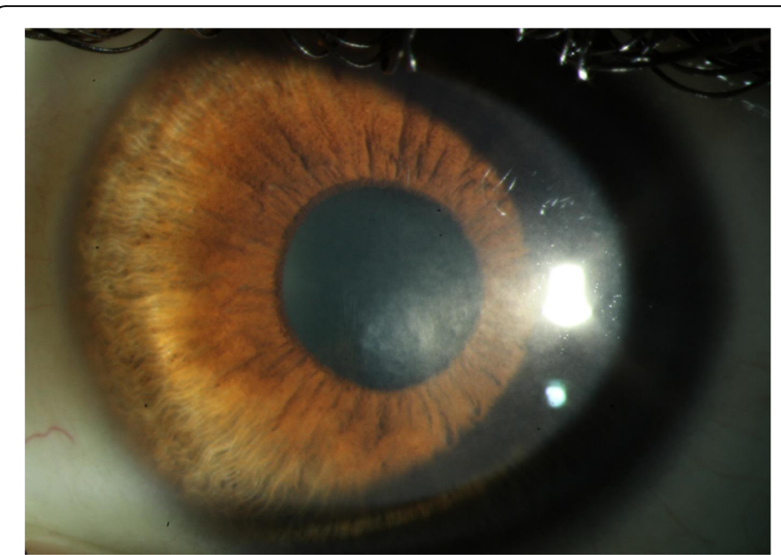

Fig. 1 Slit-lamp photograph of the left eye a few months after initial presentation. The inferonasal part of the cornea showed a hazy opacification of the deep stroma

left eye. The endothelial cell count with specular microscopy (Tomey EM-3000) was minimally reduced to 2408 cells $/ \mathrm{mm}^{2}$ in the left eye in comparison with the right eye $\left(2525\right.$ cells $\left./ \mathrm{mm}^{2}\right)$. Blood analyses showed resolved VZV and HSV infections (enzyme-linked immunosorbent assay, ELISA), resolved CMV infection (electrochemiluminescence immunoassay, ECLIA) and hepatitis B immunity (ECLIA). No active infection (incl. borrelia) or systemic inflammation could be found; thorax radiology was without pathology.
Based on the clinical diagnosis of non-necrotising stromal keratitis of presumed herpetic origin, we treated the patient with corticoid eye drops, ganciclovir eye gel and acyclovir tablets. During the 2-year follow-up of the patient, relapses occurred with reduction of the therapy, which required regular increases of the frequency of topical therapy application or temporary use of systemic steroid.

Despite regular controls and therapy adaptation, clinical examinations showed keratoconus-like changes with Vogt's striae, incomplete Fleischer Ring and signs of paracentral stroma thinning on the left eye, starting 1 year after onset of the disease. Two years after presentation, BCVA of the affected eye was reduced to 20/200; maximal anterior, posterior and true net powers had increased to $66.9,-8.1$ and $48.8 \mathrm{D}$ respectively; minimal corneal thickness decreased to $303 \mu \mathrm{m}$; and optical coherence tomography showed a cone-shaped bulge of the paracentral cornea with increased stromal hyperreflexivity (Figs. 2, 3 and 4, Table 1).

An excimer laser supported penetrating corneal transplantation was carried out at this point on the left eye.

Pathological analyses of the excised cornea showed a chronic (stromal) interstitial keratitis with stromal scarring, edema and myofibroblastic activation of stromal cell (Fig. 5a-c). In special stains (Congo red, Grocott-Gomori's, PAS, Gram) and in situ hybridization, the presence of fungus, borrelia, cytomegalie virus, herpes simplex virus (type I and II) or EBV were excluded. Three months after

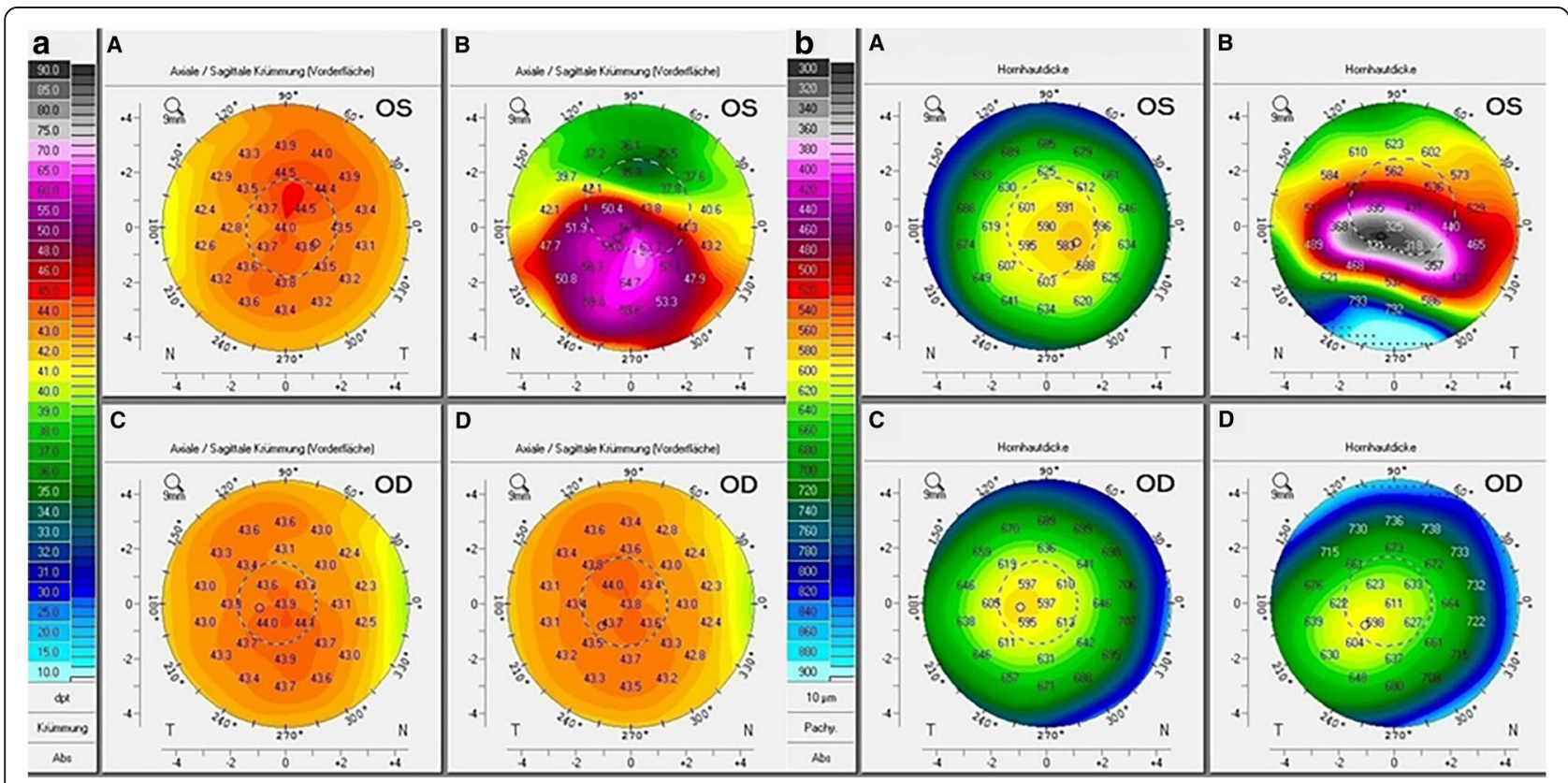

Fig. 2 Pentacam tomography showing a maximal power of the anterior surface of the left eye (OS) increased between day of presentation (A, 44.9D) and last control before corneal transplantation (B, 66.9D). b Corneal thickness of the left eye decreased between day of presentation (A, $581 \mu \mathrm{m})$ and last control before corneal transplantation (B, $303 \mu \mathrm{m})$. No changes are noticed on the right eye between day of presentation (c) and last control before corneal transplantation (d). $N$ nasal, $T$ temporal 


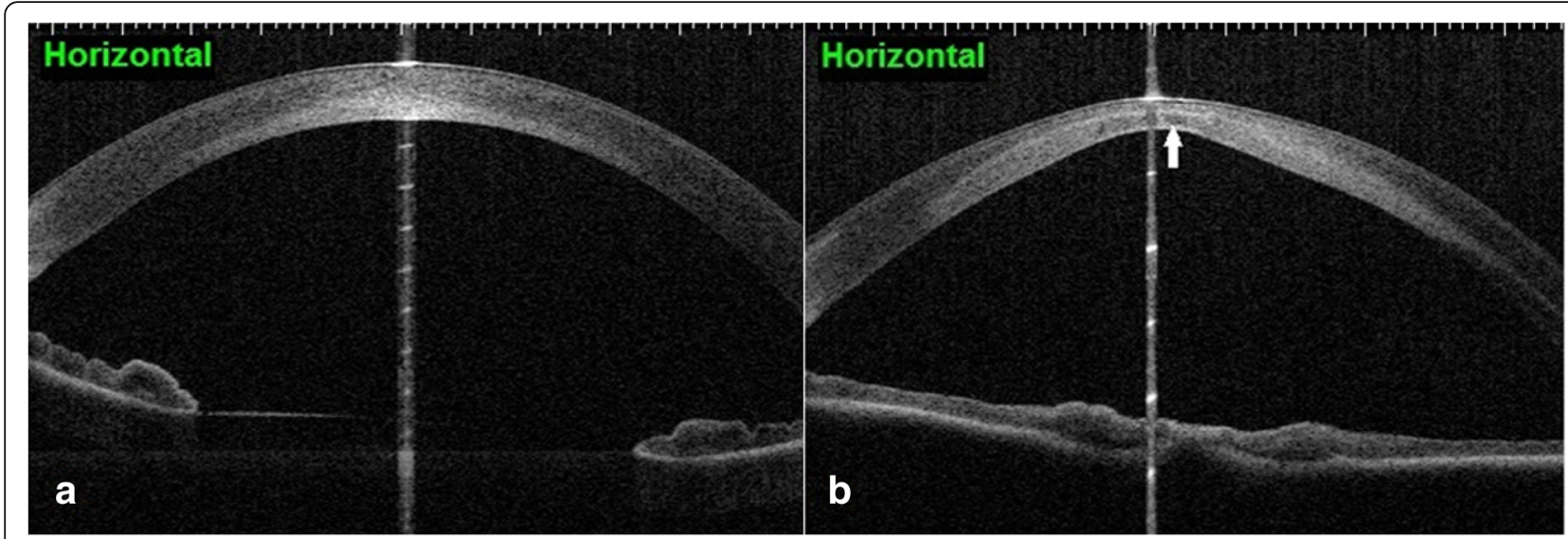

Fig. 3 Optical coherence tomography of the left eye at day of presentation (a) and at last control before keratoplasty (b) showing a cone-shaped bulge of the cornea with corneal thinning and increase of stromal hyper-reflexivity in the posterior part (arrow)

transplantation, the patient recovered a BCVA of 20/32 on the left eye and no recurrence was observed to date.

\section{Discussion}

Originally, keratoconus was presented as a noninflammatory disorder affecting primarily young men, usually bilateral and leading to visual impairment.
Although the pathogenesis of keratoconus remains poorly understood, multiple studies revealed the importance of inflammation in the progression of the disease and a recent consensus was to consider keratoconus an inflammatory disease $[3,4]$. Accordingly, novel therapeutic approaches aiming to prevent keratoconus progression through reduction of inflammation were evaluated. The topical application of immune-modulator

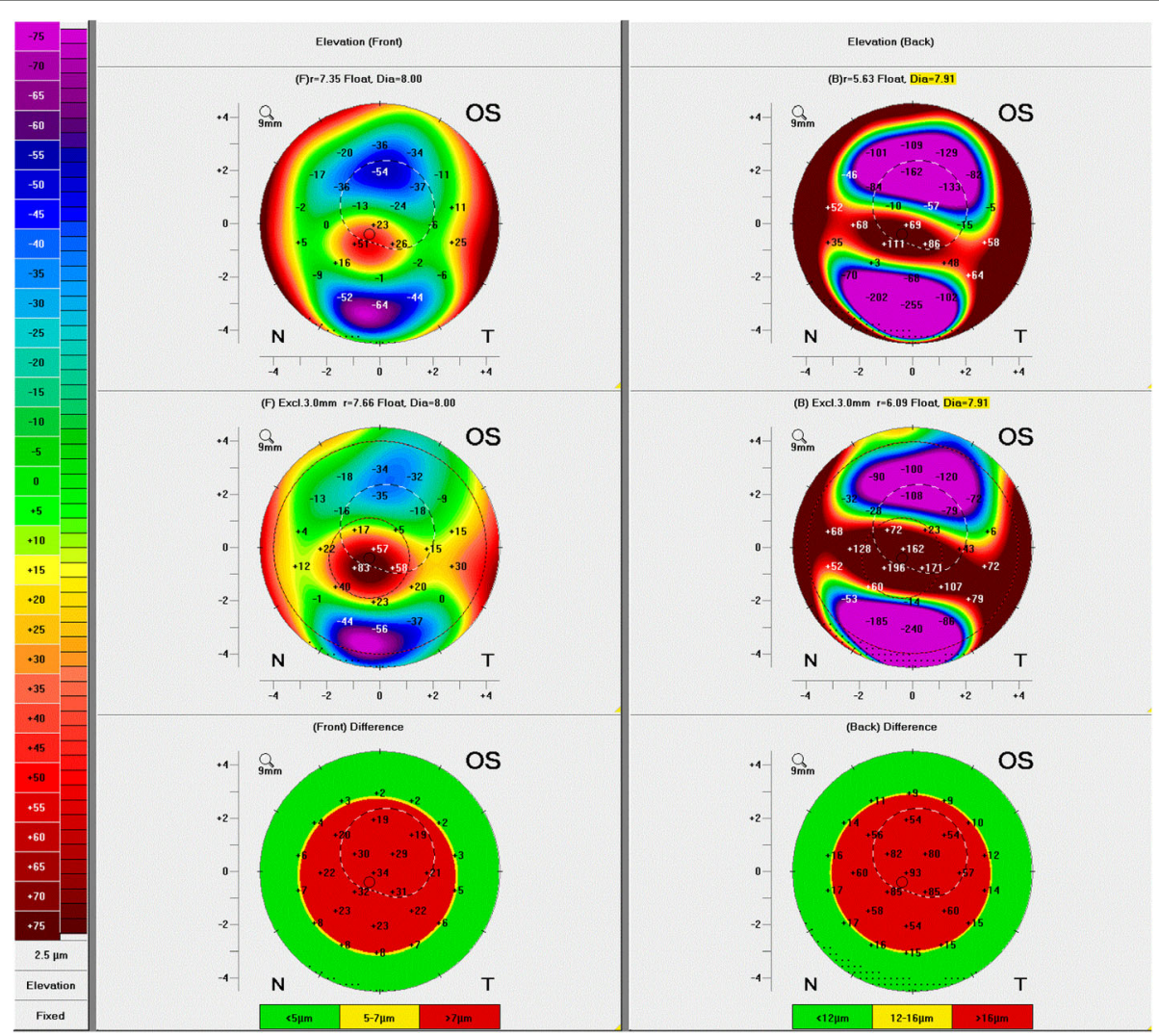

Fig. 4 Elevation maps of the anterior (left) and posterior surface (right) of the left eye before corneal transplantation 
Table 1 Evolution of the left eye

\begin{tabular}{|c|c|c|c|c|c|c|c|}
\hline & & Month 0 & Month 4 & Month 11 & Month 16 & Month 19 & Month 22 \\
\hline BCVA & & $20 / 20$ & $20 / 20$ & $20 / 20$ & $20 / 100$ & $20 / 200$ & $20 / 200$ \\
\hline Pachymetry at the thinnest point $(\mu \mathrm{m})$ & & 581 & n.a. & 511 & 427 & 360 & 303 \\
\hline \multirow[t]{3}{*}{ Power of the anterior surface (dpt) } & $\mathrm{K} 1$ & 43.3 & 43.2 & 42.7 & 44.8 & 44.2 & 49.0 \\
\hline & $\mathrm{K} 2$ & 44.4 & 44.4 & 43.2 & 46.1 & 47.0 & 52.3 \\
\hline & Kmax & 44.9 & 44.8 & 45.5 & 49.4 & 59.3 & 66.9 \\
\hline \multirow[t]{3}{*}{ Power of the posterior surface (dpt) } & $\mathrm{K} 1$ & -6.0 & -5.5 & -6.4 & -6.7 & -8.0 & -7.8 \\
\hline & $\mathrm{K} 2$ & -6.2 & -5.8 & -5.9 & -6.3 & -7.3 & -8.4 \\
\hline & Kmax & -6.1 & -5.6 & -6.1 & -6.5 & -7.6 & -8.1 \\
\hline True net power (dpt) & $\mathrm{Km}$ & 42.8 & 43.2 & 41.7 & 44.2 & 44.1 & 48.8 \\
\hline
\end{tabular}

$K 1$ power of the steep meridian, $K 2$ power of the flat meridian, Kmax maximal corneal power, Km mean keratometry measure, n.a. not available

cyclosporine A demonstrated encouraging results in a small amount of keratoconus patients [2].

Eye rubbing, atopy or contact lens wear were also revealed as risk factors in clinical and experimental studies, suggesting the role of environmental aspects [7-9]. As eye rubbing showed to be associated with increased levels of proinflammatory mediators in tears, its influence on keratoconus may be related to pro-inflammatory effect [7]. Keratoconus has also been associated with metabolic disorders of the urea cycle [10] and thyroid gland dysfunction [11]. Multiple studies support the influence of genetic components in the pathogenesis of the disease $[9,12]$.

In recent studies, the effect of infectious keratitis on corneal topography was investigated $[5,6]$. The topographic changes due to infectious keratitis seem to depend on the location of the inflammation and the healing response of the host. Although asymmetric patterns appeared to be the most common, protrusion patterns were also revealed [5]. The pathogenesis of these changes has not been elucidated yet, but the first patterns may be associated to stiffening of the inflamed cornea due to a predominance of scarring process, whereas the second, keratoconus-like patterns would arise through stromal melting. Optical aberrations caused by topographic changes of the cornea were revealed, as well as corneal opacification, to be responsible for visual impairment following infectious keratitis [5].

Our patient had no systemic disease or familial history of keratoconus. $\mathrm{He}$ developed rapid keratoconus-like corneal changes on the eye with recurrent inflammatory manifestations but no clinical signs of keratoconus in the other eye. This clinical case may support the essential role of inflammation in the development and progression of keratoconuslike ecstasies. Although the cause remains unclear, the rapidity of the progression of keratoconus in this case could be related to the age of the patient, which correlates with the usual onset of the disease.

This case demonstrates that keratitis may lead to keratoconus-like corneal changes and supports the hypothesis of a causative link between recurrent inflammatory process and the development of keratoconus. It provides additional arguments towards the use of stronger anti-inflammatory medications or immunosuppressive drugs such as cyclosporine in similar cases to prevent corneal ectasia and corneal transplantation.

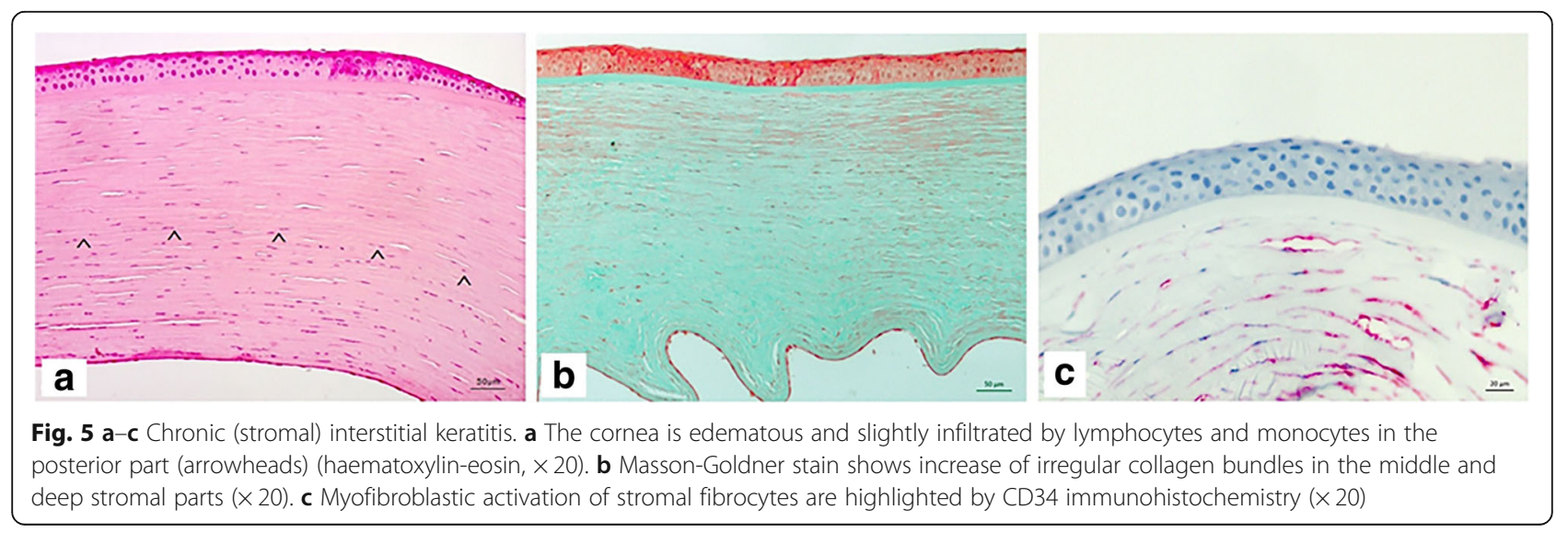




\section{Funding}

No funding sources.

\section{Authors' contributions}

MSH collected the data and wrote the main manuscript text. All authors read and approved the final manuscript.

\section{Consent for publication}

An informed consent for publication was obtained from the patient.

\section{Competing interests}

The authors declare that they have no competing interests.

\section{Publisher's Note}

Springer Nature remains neutral with regard to jurisdictional claims in published maps and institutional affiliations.

\section{Author details}

'Department of Ophthalmology, Saarland University Medical Center, 66424 Homburg, Saar, Germany. ${ }^{2}$ Institute of Pathology, Saarland University Medical Center, 66424 Homburg, Saar, Germany.

Received: 2 November 2017 Accepted: 13 February 2018

Published online: 07 March 2018

\section{References}

1. Rabinowitz YS (1998) Keratoconus. Surv Ophthalmol 42:297-319

2. Shetty R, Ghosh A, Lim RR, Subramani M, Mihir K, Reshma AR, Ranganath A, Nagaraj S, Nuijts RM, Beuerman R, Shetty R, Das D, Chaurasia SS, Sinha-Roy A, Ghosh A (2015) Elevated expression of matrix metalloproteinase-9 and inflammatory cytokines in keratoconus patients is inhibited by cyclosporine A. Invest Ophthalmol Vis Sci 56:738-750

3. Galvis V, Sherwin T, Tello A, Merayo J, Barrera R, Acera A (2015) Keratoconus: an inflammatory disorder? Eye (Lond) 29:843-859

4. Wisse RP, Kuiper JJ, Gans R, Imhof S, Radstake TR, Van der Lelij A (2015) Cytokine expression in keratoconus and its corneal microenvironment: a systematic review. Ocul Surf 13:272-283

5. Shimizu E, Yamaguchi T et al (2017) Corneal higher-order aberration in infection keratitis. Am J Ophthalmol 175:148-158

6. Kashizuka E, Yamaguchi T et al (2016) Corneal higher-order aberrations in herpes simplex keratitis. Cornea Epub ahead of prints

7. loannidis AS, Speedwell L, Nischal KK (2005) Unilateral keratoconus in a child with chronic and persistent eye rubbing. Am J Ophthalmol 139:356-357

8. Galvis V, Tello A, Carreño NI, Berrospi RD, Niño CA (2017) Risk factors for keratoconus: atopy and eye rubbing. Cornea 36:1

9. Gordon-Shaag A, Millodot M, Shneor E, Yutao L (2015) The genetic and environmental factors for keratoconus. Biomed Res Int. https://doi.org/10. 1155/2015/795738

10. Stachon T, Kolev K, Flaskó Z, Seitz B, Langenbucher A, Szentmáry N (2017) Arginase activity, urea, and hydroxyproline concentration are reduced in keratoconus keratocytes. Graefes Arch Clin Exp Ophthalmol 255:91-97

11. Thanos S, Oellers P, Zu Hörste M, Prokosch V, Schlatt S, Seitz B, Gatzioufas Z (2016) Role of thyroxine in the development of keratoconus. Cornea 35:1338-1346

12. Nowak DM, Gajecka M (2011) The genetics of keratoconus. Middle East Afr J Ophthalmol 18(1):2.

\section{Submit your manuscript to a SpringerOpen ${ }^{\circ}$ journal and benefit from:}

- Convenient online submission

- Rigorous peer review

- Open access: articles freely available online

- High visibility within the field

Retaining the copyright to your article 